and body mass index [table 1]. Given the low number of non-Caucasians we were unable to comment on the relationship between antibody and race.

Medication choice and mortality were independent of mean household income. Rituximab was the most commonly prescribed ISM [44\%]. The median age of rituximab exposure patients was 65 years and 60 years for unexposed patents. There was no significant relationship between age and medication choice. Rituximab was prescribed $60 \%$ of the time in renal AAV $[p<0.01]$ and $53 \%$ of the time in pulmonary AAV $[p<0.01]$. Other commonly prescribed medications in the cohor included Mycophenolate [20\%], Methotrexate [24\%] and Cyclophosphamide [28\%].

The overall mortality rate was $17 \%$ [13/75] [figure 1]. There was no significant difference in the mortality rate of patients 65 years and older [23\%, 9/39] compared to patients 64 and younger $[11 \%, 4 / 36 ; p=0.17]$. The mortality rate was also independent of median household income.

Abstract AB0645 - Table 1

\begin{tabular}{|c|c|c|c|c|}
\hline & \multicolumn{2}{|c|}{ PR3 antibody } & \multicolumn{2}{|c|}{ MPO antibody } \\
\hline & Negative & Positive & Negative & Positive \\
\hline BMl<29.9 & $69 \%$ & $31 \%$ & $71 \%$ & $29 \%$ \\
\hline $\begin{array}{l}\text { BMI 30- } \\
39.9\end{array}$ & $35 \%$ & $65 \%$ & $75 \%$ & $25 \%$ \\
\hline $\begin{array}{l}\mathrm{BMI}>40 \\
\mathrm{p} \text {-value }\end{array}$ & $20 \%$ & $80 \%$ & $100 \%$ & $\mathrm{~N} / \mathrm{A}$ \\
\hline
\end{tabular}

BMI: body mass index, PR3: proteinase 3, MPO: Myeloperoxidase

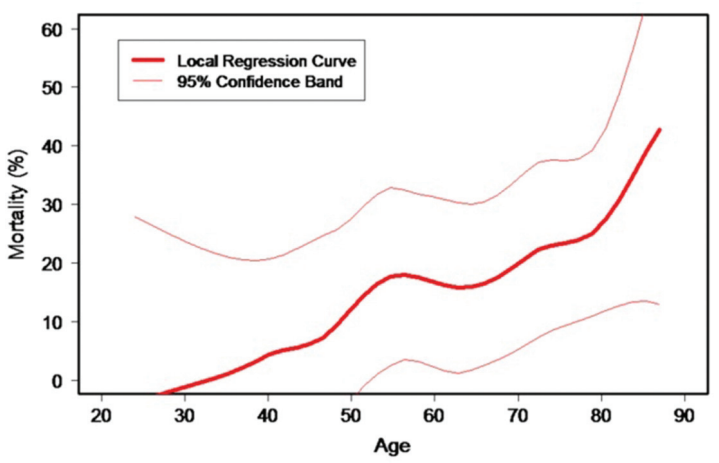

Abstract AB0645 - Figure 1

Conclusions: There was no relationship between age, sex, income, immunosuppressive therapy, and mortality in our AAV cohort. The mortality rate was higher in more severe disease and rituximab was more commonly used in such cases as well as in older patients. The limitations of the study include this being a hospital based cohort, where social demographics may have less of an impact. There was a lack of racial diversity, though the cohort did reflect the general demographics in southwest Virginia.

Disclosure of Interest: None declared

DOI: 10.1136/annrheumdis-2018-eular.1738

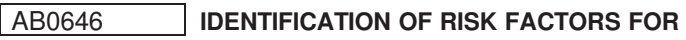 RECURRENCE IN POLYMYALGIA RHEUMATICA}

A. Ueno, M. Hirata, Y. Yamamura, K. Fujita, N. Shibutou, M. Yamamura. Center for Rheumatology, Okayama Saiseikai General Hospital, Okayama, Japan

Background: Glucocorticoids (GCs) are effective for polymyalgia rheumatica (PMR); however, some patients relapse during GC tapering and develop adverse events of GCs.

Objectives: To identify risk factors for recurrence in patients with polymyalgia Rheumatica(PMR).

Methods: Cox proportional hazards regression analyses was performed 78 patients with PMR who had been treated by 2015 EULAR/ACR recommendations between January2015 and December 2017 in our centre. The following data at baseline were collected retrospectively: age, sex, time of diagnosis, location of arthralgia/myalgia, maximum dose of prednisolone, US findings of shoulder, laboratory data before the initial treatment.

Results: Seventy-eight patients had been diagnosed with the 2012 EULAR/ACR provisional classification criteria for PMR, and had been treated first with GCs. They were at the age of $71.7 \pm 9.5$, including 34 males and 44 females. Duration of symptoms before therapy was $2.0 \pm 2.0$ months and the PMR duration was 21.2 \pm 15.5 months. Twenty-seven patients had arthralgia/myalgia other than shoulders and hips. The maximum dose of prednisolone was $15.9 \pm 4.4 \mathrm{mg} /$ day. US findings of shoulder were positive in 65 patients. Relapses occurred in 37 patients (47\%), when the dose of prednisolone was reduced to $5.6 \pm 5.5 \mathrm{mg} /$ day at $8.9 \pm 6.4$ months. MTX $(8.6 \pm 3.1 \mathrm{mg} / \mathrm{wk})$ was added in 29 patients or the dose of prednisolone was increased in 8 patients, similarly to the 2015 EULAR/ACR recommendations for the management of PMR. Additional MTX was ineffective in 16 patients followed by adding tocilizumab in 8 patients. Forty patients discontinued GCs at $17.6 \pm 10.6$ months.

On univariate analysis, 4 variables were identified as significant risk factors affecting PMR recurrence: increased platelets $(p=0.00192), \operatorname{low} \lg A \quad(p=0.00149)$, arthralgia/myalgia limited to shoulders and hips $(\mathrm{p}=0.02)$, and the maximum dose of prednisolone used ( $\mathrm{p}=0.0062$ ). These 4 variables were introduced into the multivariate analysis, and the following 3 variables were retained as independent significant risk factors: the maximum dose of prednisolone $(\mathrm{p}<0.005)$, limitation of arthralgia/myalgia to shoulders and hips $(p<0.05)$ and $\operatorname{low} \lg A(p<0.005)$

Conclusions: These results indicate that the maximum dose of prednisolone, the absence of peripheral joint pains and low $\lg A$ may be associated with the recurrence in PMR patients

Disclosure of Interest: None declared

DOI: 10.1136/annrheumdis-2018-eular.7128

\section{AB0647 TAKAYASU'S ARTERITIS IN ITALY: CLINICAL PRESENTATION, DIAGNOSTIC DELAY AND VASCULAR PATTERN}

A. Tomelleri, C. Campochiaro, S. Sartorelli, C. Sembenini, S. Franchini, F. Motta, D. Vanni, G. Cavalli, E. Baldissera, L. Dagna. Unit of Rheumatology, Immunology, Allergy and Rare Diseases (UnIRAR), San Raffaele Scientific Institute, Milan, Italy

Background: Takayasu arteritis(TA) is a large vessel vasculitis ${ }^{1}$. At disease presentation TA patients may present with inflammatory symptoms, sometimes associated with vascular pain. As arterial lesions ensue, more characteristic features may be found, such as limb claudication, decreased or absent pulses, vascular bruits, discrepancies in blood pressure ${ }^{2}$. Since initial symptoms are often nonspecific, early detection of TA is challenging and patients at diagnosis frequently have established damage ${ }^{3}$

Objectives: To describe symptoms presentation and vascular involvement in a large cohort of Italian TA patients. To assess the diagnostic delay associated with symptoms and arteries involved at presentation

Methods: Data from 114 TA patients diagnosed according to ACR classification criteria at our tertiary centre were retrospectively collected. Signs and symptoms subsequently attributed to TA, age at first symptom onset, and age at diagnosis were analysed. The diagnostic delay was calculated. Arteries involved before the diagnosis were identified. Non-parametric statistic tests were used

Results: The cohort included mostly female patients(104;91.2\%). Mean age at first symptom subsequently attributed to TA was 30.5 years $( \pm 13.1)$. Age at pre sentation of first TA related symptom was 18-40 years in $68(59.6),<18$ years in $20(17.5 \%),>40$ years in $26(22.8 \%)$ patients. Mean age at diagnosis was 36 years $( \pm 12.7)$. Mean diagnostic delay was 65.6 months $( \pm 85.3)$. Diagnostic delay was $>1$ year in 75 patients(65.8\%). The most frequent finding before diagnosis was raised inflammatory markers(82.4\%), the least common pulmonary hypertension $(2.8 \%)$. The features significantly associated with a diagnostic delay $>1$ year were arthralgias, hypertension, previous vascular surgery. The mean diagnostic delay (in months) was significantly higher in patients with raised inflammatory markers (8.4 \pm 4.9 vs $100.5 \pm 121.8, p=0.005)$, carotidynia( $5.8 \pm 5.7$ vs $34.2 \pm 32.9, p=0.034)$ and in patients who had upper limb claudication(11.0 \pm 13.2 vs $65.44 \pm 77.01$, $\mathrm{p}=0.002)$. Data about arteries involved before diagnosis were available for 86 patients $(75.4 \%)$. They were: subclavian in $43(50 \%)$, carotid in $38(44.2 \%)$, renal in $19(22.1 \%)$, abdominal aorta in $18(20.9 \%)$, ascending aorta in $15(17.4 \%)$, axillary in $12(14 \%)$, vertebral in $11(12.8 \%)$, superior mesenteric in $10(11.6 \%)$, thoracic aorta in $10(11.6 \%)$, aortic arch in $9(10.5 \%)$, celiac tripod in $9(10.5 \%)$, brachiocephalic in $8(9.3 \%)$, iliac in $8(9.3 \%)$, coronary in $6(7 \%)$, femoral in 6 $(7 \%)$, pulmonary in $5(5.8 \%)$, brachial in $3(3.5 \%)$, popliteal in $2(2.3 \%)$ patients. There was no significant difference in the arteries involved between patients diagnosed $<1$ year and $>1$ year from symptoms onset. Mean diagnostic delay was not significantly different according to the arteries involved

Conclusions: In Italy TA diagnosis is burdened with an important diagnostic delay. In our cohort it seems that the presence of both specific and non-specific symptoms is associated with delayed recognition of TA. Moreover, the need for vascular surgery in young women should rise the concern about TA

\section{REFERENCES}

[1] Weyand, C. M. \& Goronzy, J. J. Medium- and large-vessel vasculitis. N. Engl. J. Med. 349, 160-9 (2003)

[2] Kerr, G. S. et al. Takayasu arteritis. Ann. Intern. Med. 120, 919-29 (1994).

[3] Numano, F., Okawara, M., Inomata, H. \& Kobayashi, Y. Takayasu's arteritis. Lancet 356, 1023-1025 (2000) 